
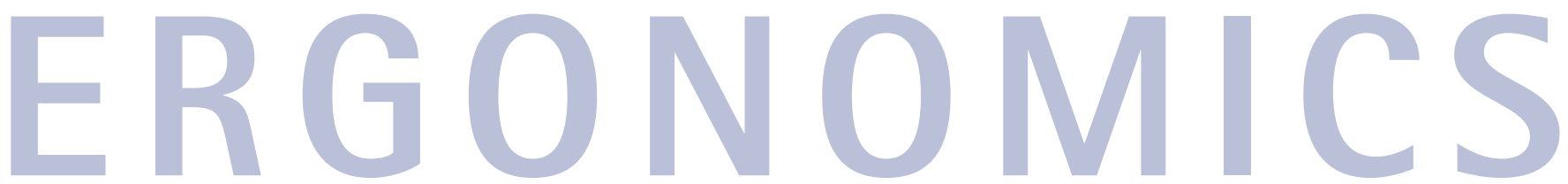

\section{Comfort first}

Support Stools claim to alleviate problems such as back, shoulder and neck ache, symptoms frequently experienced by dentists and their staff who spend large parts of their working hours concentrating whilst leaning forward with their hands stretched out in front of them.

This posture puts immense pressure on their lower back, neck and shoulder. The stools are fully adjustable, and are designed to hold the dentist in the correct anatomical posture with their pelvis tilted forward whilst allowing their spine to hold its natural ' $S$ ' shape at all times, even when leaning forward.

With their anatomically shaped seats and backs and free floating action, they follow the dentist's movements to and fro. This reduces the pressure on the spine and thus the risk of musculo-skeletal injury. It also has a range of optional accessories such as the 'Relax' armrest which holds the operators mirror hand in place whilst also allowing freedom of movement and support. Similarly the 'Hydro' armrest allows flexibility of movement for the instrument hand whilst supporting their arm. This can aid the dentist during precision procedures where long periods can be spent bending over a patient, staying relatively still in order to perform intricate manoeuvres.

Reader response number 55

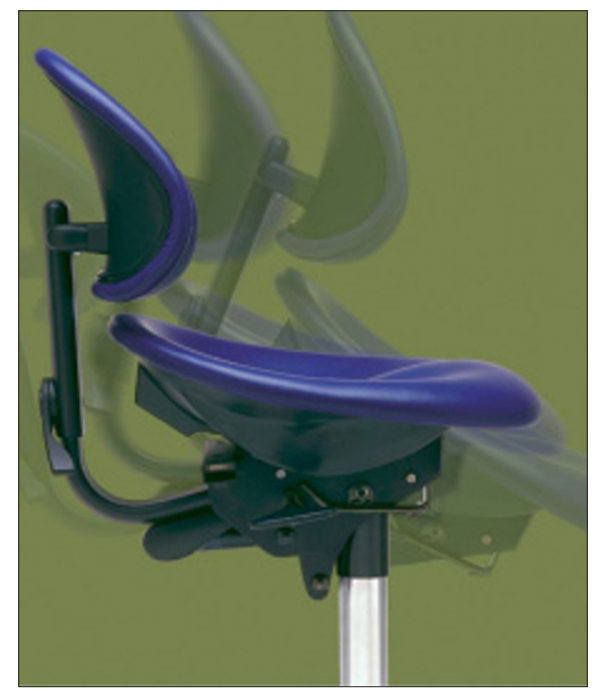

High tech treatment

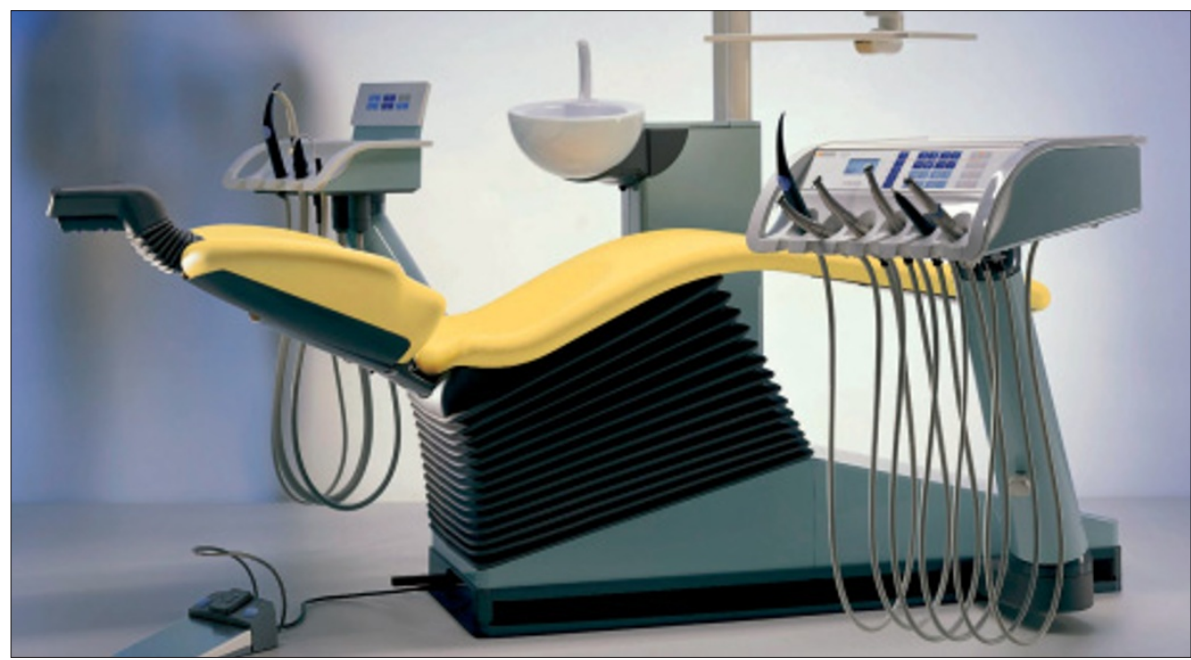

The $\mathrm{C} 2+$ from Sirona encompasses features including 'hands-on' via control keys or 'hands-free' via cursor and foot switch.

Laid out in separate blocks for chair adjustment, chair programming, handpiece function and speed, the layout and colour coding of the control keys are selfexplanatory. While in its 'hands-free' mode the $\mathrm{C} 2+$ automatically anticipates the next logical step in the treatment sequence. This function is activated by moving the cursor horizontally.

This includes a swivelling cuspidor bowl, which makes rinsing quicker and easier, a motor driven headrest, shifting side track dentist element and ErgoMotion. Sirona's ErgoMotion system ensures that movements of the backrest and seat are synchronised and follow the natural

\section{Standing out}

The new Hejco 2003 catalogue has added strong colours in the latest styles for a more casual look for staff in dental practices. Unisex shirts which are 50\% polyester and $50 \%$ cotton and ladies blouses are few of the many uniforms available in the 'Add Colour to your practice' range. All uniforms in this range are easy to care for and are machine washable up to 85 degrees. Reader response number 57 anatomical movements of the body.

Ensuring anatomically correct patient positioning for maximum comfort, this means the patient does not feel constrained or uncomfortable when the chair is repositioned. For maximum efficiency, both the dentist and assistant have easy access to the controls on the headrest, enabling them to select all the chair's functions without interrupting the treatment process.

Other ergonomic features include arrangement of the handpieces at convenient angles to ensure optimum and safe handling. Finally the anatomically shaped of the dentist stool encourage ergonomically correct posture. The seat cushion and backrest are height adjustable.

Reader response number 56

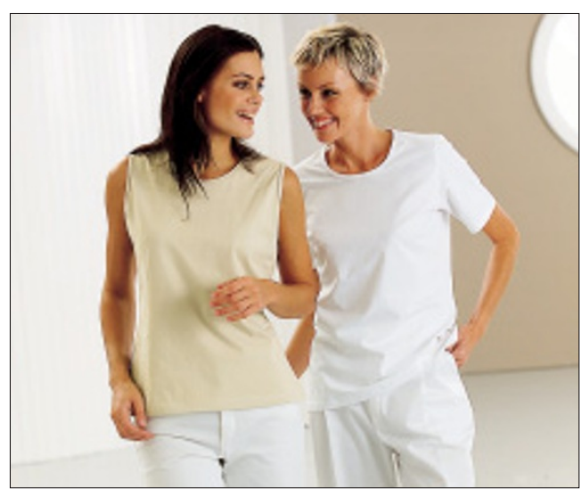
cushion and relax function of the backrest 


\section{Handy storage}

Versafile mobile cabinets are composed of modules consisting of up to four different drawer sizes to meet the storage needs of any individual or department.

The units can be constructed to the required height and width and can incorporate any mix of drawer sizes. The system combines style with proven durability making it a highly cost effective alternative to traditional, less flexible storage. Versafile is manufactured with a combination of ABS, steel and high qual-

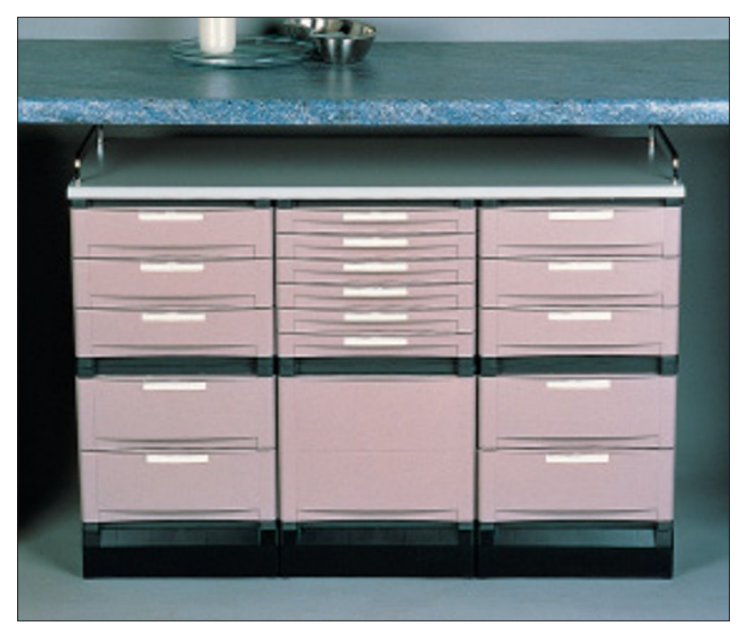
ity plastic making it easy to clean and resistant to staining. Additional extra options include lockable cupboards, drawer locks, instrument trays and drawer dividers. For wall to wall storage solutions the Versafile static system can be installed on site to provide storage in any space available.

Reader response number 58

\section{Versatility meets comfort}

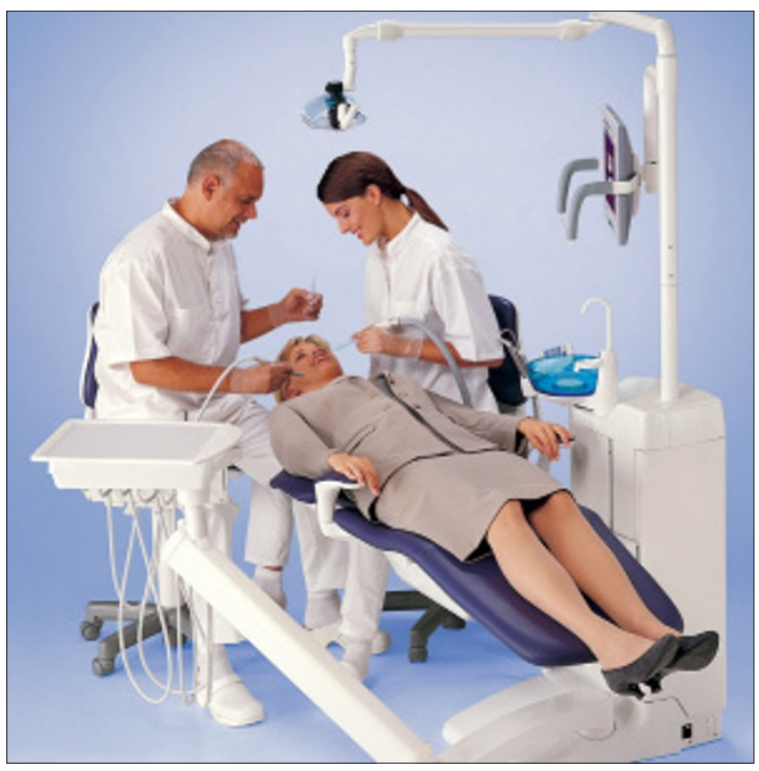

The latest addition to the Planmeca product family is the Planmeca chair, a patient chair which combines the dental team's working ergonomics and patient comfort.

The lifting mechanism is designed to maximise legroom underneath the chair and features a slim backrest to contribute to optimized working postures and easy access to the oral cavity for both right handed and left handed users. The chair is operated with a multi-functional chairbased foot control, which is easily accessible for the left and the right handed.

It can also be equipped with moveable arm rests and an automatic synchronised legrest. Other features include a needle bearing mounting and a foot controlled mechanism which contributes to better asepsis. Manual trim adjustments and automatic preprogrammed working positions are combined in one control. It can also be equipped with a 180-degree swivel which provides freedom in operatory design while the contoured upholstery conforms to the patient's anatomy. The double articulated headrest allows comfortable positioning of the head and provides clear visibility into the oral cavity.

\section{Reader response number 59}

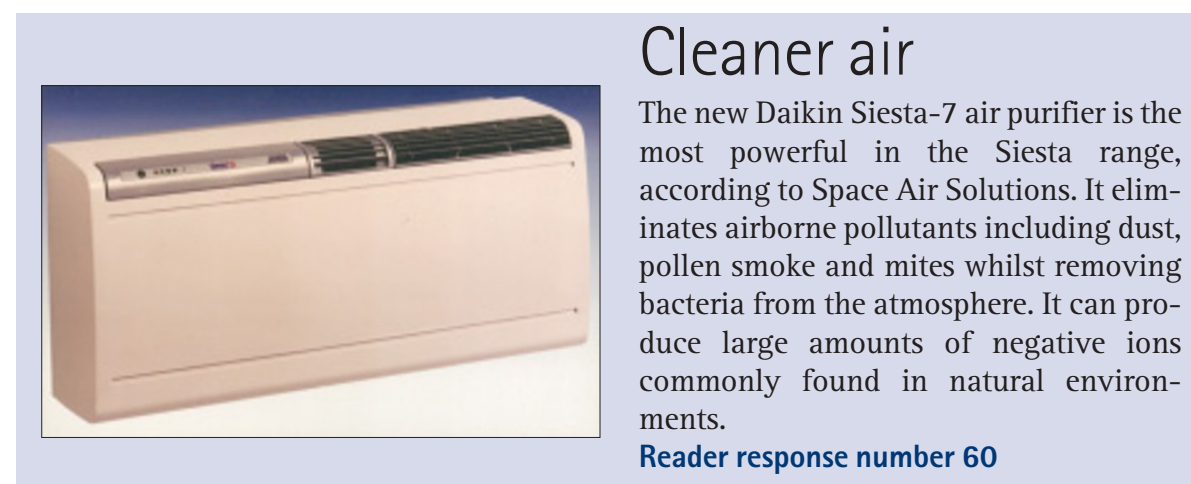

